\title{
Comparing Approaches for Capturing Bacterial Assemblages Associated with Symptomatic (Bacterial Blotch) and Asymptomatic Mushroom (Agaricus bisporus) Caps
}

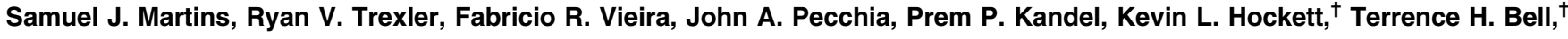 \\ and Carolee T. Bull \\ Department of Plant Pathology and Environmental Microbiology, The Pennsylvania State University, University Park, PA
}

Accepted for publication 16 October 2019.

\section{ABSTRACT}

Bacterial blotch is a complex of diseases, caused by Pseudomonas tolaasii and other Pseudomonas spp., whose growth may be influenced by other microorganisms associated with mushroom caps. The objectives of this work were to (i) compare methods for extracting DNA from mushroom caps to assess bacterial composition, and (ii) describe and quantify the composition of bacteria in and on symptomatic and asymptomatic mushroom caps. Symptomatic and asymptomatic mushrooms were collected at an organic farm in Pennsylvania and processed. Bacteria were removed from mushrooms by sonication to target surface-associated bacteria (washate), from whole mushrooms postsonication to target bacteria within or tightly adhering to the mushrooms (post-washate), or from whole macerated mushrooms to target all mushroom-associated bacteria in one sample (whole). Bacterial composition was determined through sequencing the $\mathrm{V} 4$ hypervariable region of the 16S rRNA gene on an Illumina MiSeq. Bacterial composition differed significantly based on mushroom health status and community removal method. Regardless of sampling approach, Proteobacteria and Bacteroidetes appeared as the most dominant phyla. In comparing the three extraction methods, higher Shannon diversity and Pielou's evenness were observed for the washate and post-washate methods compared with whole mushroom sampling. Additionally, higher Shannon diversity, operational taxonomic unit richness, and Pielou's evenness were found for the asymptomatic mushrooms when compared with symptomatic mushrooms. Pseudomonas spp. were overrepresented on mushroom surfaces (washate method), regardless of health status. This research made strides in describing the mushroom cap microbiome and established sampling methods that will allow researchers to target studies and isolation of specific bacterial taxa.

Keywords: 16S rRNA gene, Agaricus bisporus, bacteriology, biological control, blotch disease, microbiome, plant pathology, Pseudomonas
Agaricus bisporus, known as the white button mushroom, is the most cultivated mushroom in the Americas, Australasia, and parts of Europe (Chang and Wasser 2017; Royse et al. 2017). Interactions with bacteria are an essential feature of $A$. bisporus cultivation and

${ }^{\dagger}$ Corresponding authors: K. L. Hockett; klh450@psu.edu, and T. H. Bell; thb15@psu.edu

Funding: Financial assistance was provided by OREI (404-49 75XB) accession 1013023 PEN07065 and the Schreyer Institute for Teaching Excellence at The Pennsylvania State University. We also thank the Agricultural Experiment Station for funding (J. Pecchia and Beyer accession 1006639 PEN04589, K. Hockett accession 101571 PEN04648, and T. H. Bell accession 1016233 PEN04651).

*The $e$-Xtra logo stands for "electronic extra" and indicates that one supplementary table is published online.

The author(s) declare no conflict of interest.

(C) 2020 The American Phytopathological Society can impact mushroom development and health, particularly when those bacteria are pathogenic (Kertesz and Thai 2018). Bacterial blotch of $A$. bisporus, a complex of diseases that results in a rapid browning or blotching and deterioration of mushroom caps, is one of the most economically important diseases or disease complexes in A. bisporus production (Navarro et al. 2018; Aslani et al. 2018). These diseases are caused by at least 14 species of fluorescent Pseudomonas (members of the phylum Proteobacteria), including P. tolaasii, which has been studied for over 100 years (S. Martins et al., unpublished data) (Paine 1919; Tolaas 1915). The disease(s) can develop rapidly, in part, because mushroom tissue provides an ideal environment for bacterial growth and proliferation, because tissue moisture content is high (water activity values typically $\geq 0.98$ ) and tissue $\mathrm{pH}$ is often near neutral (MartinezCarrera et al. 1998; Rossouw and Korsten 2017; Venturini et al. 2011). Inoculation of pathogenic bacterial strains on A. bisporus caps can lead to rapid disease development within approximately 15 h (S. Martins et al., unpublished data). Recently, Navarro et al. 
(2018) estimated reduced yields in Spanish crops of A. bisporus up to $15 \%$ caused by species of Pseudomonas, including P. tolaasii. Several previous reports targeted the isolation and molecular identification of Pseudomonas species (Godfrey et al. 2001; Munsch et al. 2002; Navarro et al. 2018; Siyoum et al. 2016; Tolaas 1915) (S. Martins et al. unpublished data). These studies applied culture-dependent methods to identify Pseudomonas spp. associated with blotch disease in A. bisporus.

In contrast, some Pseudomonas spp. are beneficial to A. bisporus growth. For instance, fluorescent $P$. putida, commonly found in compost and casing layers, is critical for initiating A. bisporus fruiting bodies (Noble et al. 2003; Zarenejad et al. 2012). Additionally, many other Pseudomonas spp., including P. putida, $P$. fluorescens, P. brassicacearum, P. chlororaphis, P. koreensis, and $P$. taiwanensis have been evaluated for blotch control, resulting in almost 100\% pathogen and blotch inhibition (Aslani et al. 2018; Tajalipour et al. 2014).

Despite progress in methods to characterize cultivable Pseudomonas species, the composition of bacteria, and specifically pseudomonads, associated with mushroom caps remains unclear, as does the impact of these organisms on blotch and ultimately mushroom health and development. Determining the composition of bacteria in mushrooms with and without blotch symptoms should allow us to identify taxa that potentially impact blotch development, either positively or negatively. Moreover, because some species of Pseudomonas promote mushroom development while others cause disease (Osdaghi et al. 2019), it is important to specifically target pathogenic pseudomonads without inhibiting those that modulate disease control and/or mushroom development. To our knowledge, no published research has compared approaches for examining the microbiome associated with $A$. bisporus tissues. Understanding the impact of different sampling methods for assessing mushroom cap-associated bacterial composition will aid researchers in understanding the intermicrobial dynamics underlying mushroom blotch emergence or its prevention. Moreover, information about the bacteria present on asymptomatic and symptomatic mushrooms would allow us to differentiate the species present in each of the two environments as potential organisms for use in biological control.

In this project, we collected mushrooms with and without blotch symptoms from a commercial organic farm in Pennsylvania and characterized bacterial composition using three culture-independent sampling methods, followed by sequencing of the amplified 16S rRNA gene region from extracted DNA. We aimed to determine (i) which sampling approach best differentiates bacterial assemblages associated with symptomatic and asymptomatic mushrooms, and (ii) whether specific bacteria are consistently associated with mushrooms with different visible health status.

\section{MATERIALS AND METHODS}

Mushroom sampling, processing, and bacterial extraction. Mushrooms were collected from an organic mushroom farm located in Blandon, Pennsylvania, U.S.A. A total of 100 mature mushrooms were collected: 50 mushrooms demonstrated blotch symptoms and 50 were asymptomatic. This experiment was conducted once. All mushrooms collected were located in the same room, thus sampled under same the environmental conditions $\left(\mathrm{ca} .17^{\circ} \mathrm{C} ; \mathrm{CO}_{2}\right.$ minimum $1,500 \mathrm{ppm}$, maximum 1,600 ppm). The mushrooms were collected from locations (primarily on the second bed from the floor) where blotch was observed, and asymptomatic and symptomatic mushrooms were always collected in pairs, with a distance of no more than $60 \mathrm{~cm}$ from each other. For each pair collection, healthy mushroom was collected first and gloved hands were sanitized with $70 \%$ ethanol between the harvesting of each mushroom. Individual mushrooms were placed in sterile 118-ml specimen cups, closed with a screw lid and labeled. The cups containing samples were placed in a container with ice and transported to the Department of Plant Pathology and Environmental Microbiology at Pennsylvania State University in University Park, Pennsylvania, where sample processing was performed.

In order to evaluate different components of the mushroom cap microbiome, one of three methods were used to collect, respectively, lightly adhering surface-associated bacteria ("washate"), bacteria within or tightly adhering to mushroom caps ("postwashate"), or the entire community ("whole"). Initial collection involved either sonication (washate), maceration after sonication (post-washate), or direct maceration of unwashed mushrooms (whole), each followed by downstream DNA extraction (Fig. 1). All mushroom caps were initially weighed. For washate sampling, $80 \mathrm{ml}$ of $10 \mathrm{mM}$ phosphate buffer ( $\mathrm{pH}$ 7.0) was added to surround each designated mushroom cap, and samples were sonicated for $5 \mathrm{~min}$ at $40 \mathrm{kHz}$ in an Ultrasonic bath (Branson Ultrasonic Cleaner, Model 8800, Branson Ultrasonics Corp., Danbury, CT, U.S.A.). The resulting washate was used for DNA extraction. Sonicated mushrooms were then transferred to sterile stomacher plastic bags with $25 \mathrm{ml}$ of $10 \mathrm{mM}$ phosphate buffer ( $\mathrm{pH}$ 7.0) and macerated for 30 s using the Seward Stomacher Model 400C Circulator. The macerated mushrooms were then used for DNA extractions (postwashate). For 50 mushrooms (25 symptomatic and 25 asymptomatic), the washate and post-washate samples were obtained from the same mushroom. For a separate 50 mushrooms ( 25 symptomatic and 25 asymptomatic), whole mushrooms were directly macerated in $25 \mathrm{ml}$ of $10 \mathrm{mM}$ phosphate buffer ( $\mathrm{pH} 7.0$ ), as described previously, with no prior sonication, and the resulting biomass was stored for DNA extractions (whole method).

DNA extractions for the washate were performed using the MoBio PowerWater DNA Extraction kit (Hilden, Germany). The washate resulting from sonication was transferred onto a $0.2 \mu \mathrm{m}$ filter (Pall Life Sciences, \#4803) by vacuum and the walls of the collection filter vessel were washed with $60 \mathrm{ml}$ of sterile buffer. The filters were removed with sterile forceps and extracted according to the manufacturer's directions. DNA extractions for samples obtained through the other two collection methods were extracted using Nucleospin 96-Soil Kits (Macherey-Nagel, Bethlehem, PA, U.S.A.). The samples containing macerated mushroom tissue resulting from the post-washate and whole methods were directly transferred into the extraction tubes $(\sim 100 \mathrm{mg})$ and further extracted as suggested by the manufacturer.

PCR amplification and sequencing. A two-step amplification and dual-barcoding approach (Kozich et al. 2013) was performed to generate Illumina-compatible amplicons (R. Trexler and T. Bell, unpublished data). Specifically, we used the universal bacterial primer set 515F (5'-GTGYCAGCMGCCGCGGTAA-3') and 806R (5'-GGACTACNVGGGTWTCTAAT-3') to amplify the v4 region of the 16S rRNA gene (Apprill et al. 2015; Parada et al. 2016). PCR conditions were as follows: $8 \mu \mathrm{l}$ of 5Prime HotStart MasterMix (Quanta BioSciences Inc., Beverly, MA, U.S.A.), 0.1 to $1 \mu \mathrm{l}$ of template DNA, $1 \mu \mathrm{l}$ of each primer from $10 \mu \mathrm{M}$ stocks, and the appropriate volume of molecular biology grade water to bring the reaction to $20 \mu \mathrm{l}$. Thermal cycling was carried out on Mastercycler Nexus Gradient PCR machines (Eppendorf, Hamburg, Germany) under the following conditions: $94^{\circ} \mathrm{C}$ for $3 \mathrm{~min} ; 25$ cycles of $94^{\circ} \mathrm{C}$ for $45 \mathrm{~s}, 50^{\circ} \mathrm{C}$ for $60 \mathrm{~s}$, and $72^{\circ} \mathrm{C}$ for $90 \mathrm{~s}$; followed by a final elongation for $10 \mathrm{~min}$ at $72^{\circ} \mathrm{C}$.

The resulting amplicons were cleaned using Mag-Bind TotalPure NGS (Omega Bio-Tek, Norcross, GA, U.S.A.) magnetic beads. The target gene primers were designed with overhang regions for a secondary PCR to attach the barcodes and standard Illumina 
adapters, and accordingly $5 \mu \mathrm{l}$ of cleaned PCR product, $12.5 \mu \mathrm{l}$ of 5Prime Hotstart Mastermix, $2.5 \mu \mathrm{l}$ of water, and $2.5 \mu \mathrm{l}$ of each index primer $(10 \mu \mathrm{M})$ were added to each reaction and amplified under the following conditions: $98^{\circ} \mathrm{C}$ for $1 \mathrm{~min}$; eight cycles of $98^{\circ} \mathrm{C}$ for $15 \mathrm{~s}$, $55^{\circ} \mathrm{C}$ for $30 \mathrm{~s}$, and $72^{\circ} \mathrm{C}$ for $20 \mathrm{~s}$; followed by a final elongation for $5 \mathrm{~min}$ at $72^{\circ} \mathrm{C}$. Barcoded amplicons were then normalized to $25 \mathrm{ng} /$ sample using the SequalPrep Normalization Plate Kit (Invitrogen, Carlsbad, CA, U.S.A.), pooled, and concentrated on a Savant SpeedVac (Thermo Scientific, Waltham, MA, U.S.A.) at $50^{\circ} \mathrm{C}$ for $3 \mathrm{~h}$. Half of the pool (12.5 ng/sample) was then purified via agarose gel extraction with the PureLink Quick Gel Extraction kit (Invitrogen) and sent to the Cornell University Biotechnology Resource Center Genomics Facility where it was sequenced on an Illumina MiSeq using the $2 \times 250$ cycle v2 kit according to the manufacturer's recommendations. After quality filtering, we obtained $10,008,086$ total reads across 148 samples, providing an average of 67,622 reads/sample. Raw read data were submitted to the NCBI SRA under the accession number PRJNA532639.

Initial data processing. Read processing and data analysis was completed (Howard et al. 2017) (R. Trexler and T. Bell, unpublished data). Here, Mothur v1.39.5 (Schloss et al. 2009) was used to merge forward and reverse reads and trim primer sequences ( diffs $=2$, maxambig $=0$ ). After removing singletons, reads were split into single fastq files for each sample and the appropriate qiime labels were added using MacQIIME v1.9.1 (Caporaso et al. 2010) before concatenating back into a single data file. A modified Brazilian Microbiome Project pipeline was utilized for operational taxonomic unit (OTU) clustering (Pylro et al. 2014). Specifically, USEARCH 7 (Edgar 2010) was used to dereplicate sequences and cluster OTUs at $97 \%$ similarity. OTU taxonomy was assigned in Mothur using the greengenes May 2013 database (McDonald et al. 2012) and OTUs classified as Archaea, Eukaryota, Plantae, chloroplast, mitochondria, and 'unknown' were removed from the dataset.

Further analysis was performed on the R software (version 3.6.1). Samples were rarified to 1900 reads/sample and those falling below this threshold were removed from the dataset. Bray-Curtis dissimilarities, nonmetric multidimensional scaling, alpha diversity measures, and permutational analysis of variance tests were performed using the 'vegan' $\mathrm{R}$ package (Oksanen et al. 2015). Community composition was analyzed using the 'phyloseq' (McMurdie and Holmes 2013) and 'metacoder' (Foster et al. 2016) $\mathrm{R}$ packages. For the metacoder R package, OTUs of the bacterial community between symptomatic and asymptomatic mushroom caps were compared, considering all the bacterial extraction methods. Differences in taxonomic distributions between extraction methods were tested using two-sided Student's $t$ test with the 'stats' $\mathrm{R}$ package. Graphs and figures were further refined using Adobe Illustrator CC 2018.

Comparison of sequences to Pseudomonas type strains and reference strains for unnamed Pseudomonas spp. Because Pseudomonas spp. are important members of the mushroom microbiome and devome, we attempted to more finely identify Pseudomonas spp. in these samples using exact sequence variants

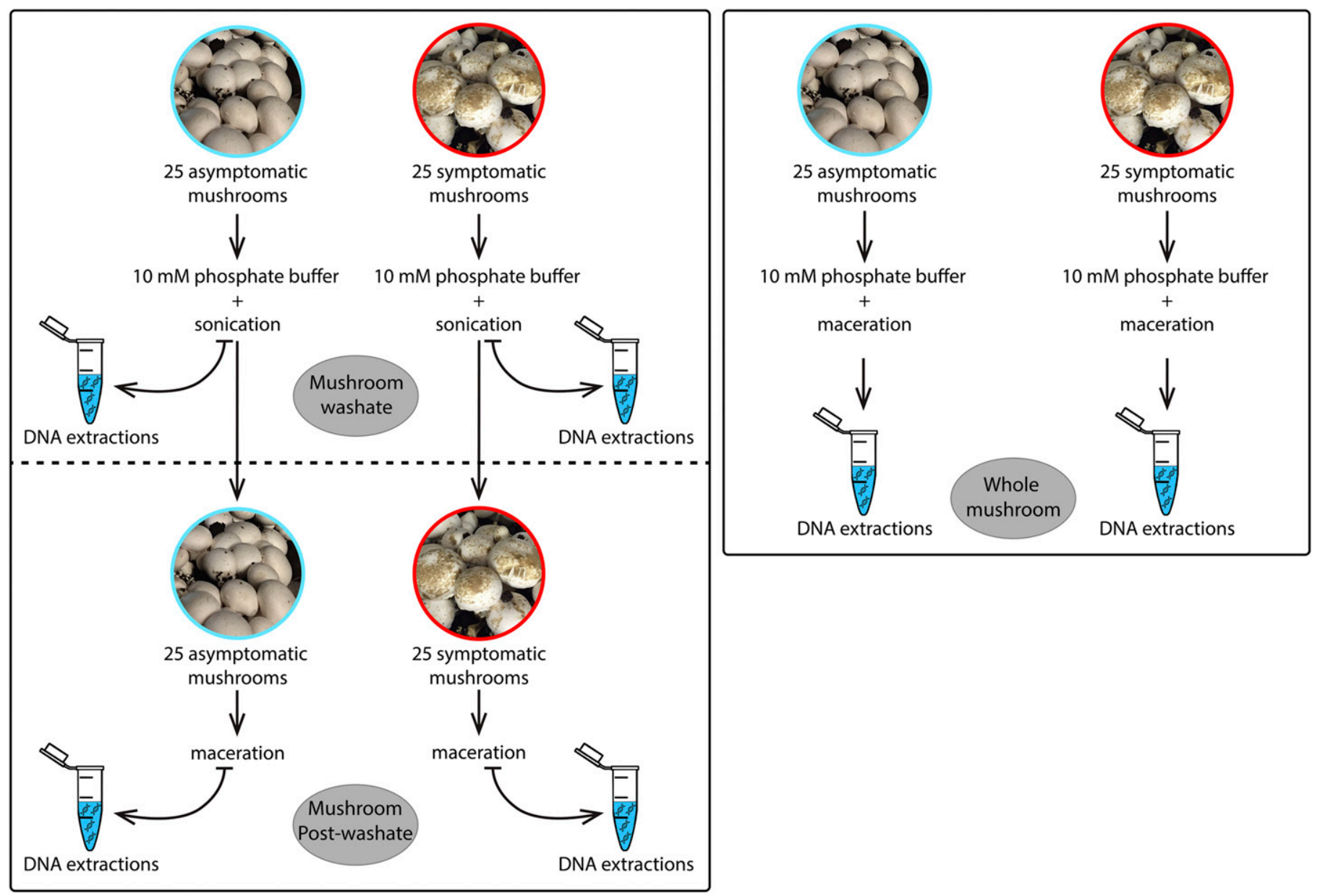

Fig. 1. Scheme of the methods for the isolation of bacteria from asymptomatic and symptomatic mushrooms (Agaricus bisporus) collected from an organic farm located in Pennsylvania. 
(ESVs). For the purpose of including sequences classified as Pseudomonas in a phylogenetic tree alongside cultured representatives, we reprocessed our data using the DADA2 pipeline (Callahan et al. 2016) to ESVs. We primarily followed the DADA2 Pipeline Tutorial 1.6: https://benjjneb.github.io/dada2/tutorial_ 1_6.html. ESVs identified as Pseudomonas spp. were compared with 162 Pseudomonas spp. type strains and reference strains of 120 putative Pseudomonas spp. published previously (Hesse et al. 2018) and 27 previously cultured blotch-causing bacteria isolated from mushroom (S. Martins et al., unpublished data).

After assessing forward and reverse read quality (command 'plotQualityProfile'), we trimmed our sequences and removed primers with the command 'filterAndTrim' using the following parameters: truncLen $=c(240,160)$, trimLeft $=c(19,20), \operatorname{maxN}=0$, $\operatorname{maxEE}=c(2,2)$, truncQ $=2$, rm.phix $=$ TRUE, compress $=$ TRUE, multithread $=$ TRUE. We then determined the run-specific error rate with the command 'learnErrors', dereplicated sequence (command 'derepFastq'), and used the DADA2 algorithm to infer true sequence variants in forward and reverse reads (command 'dada'). Forward and reverse reads were then merged (command 'mergePairs') and assumed chimeras removed (command 'removeBimeraDenovo', method="consensus"). Taxonomy was assigned with the command 'assignTaxonomy' using the Silva database v.128 (silva_species_ assignment_v128.fa) (Quast et al. 2012).

To determine which ESVs that classified as Pseudomonas were significantly different in relative abundance between symptomatic and asymptomatic mushrooms, we performed differential abundance analysis using the DESeq2 (v.1.23.10) and phyloseq (v.1.27.6) R packages (Love et al. 2014; McMurdie and Holmes $2013,2014)$. These analyses were performed on sequence data that had not been normalized through subsampling.

First, relative abundance data, taxonomic data, and metadata were combined into a phyloseq object. We then separated the object by sampling method (i.e., wash, mushroom interior, and whole mushroom), and performed downstream DESeq2 analyses separately for each method. We used the 'phyloseq_to_deseq2' command followed by the 'DESeq' command (test = "Wald", fitType = "parametric") to test for ESVs that differed significantly in relative abundance between symptomatic and asymptomatic mushrooms. We then subselected ESVs with an adjusted $P$ value less than 0.01, and extracted only those classified as Pseudomonas. These were then used to generate and annotate the phylogenetic tree, using neighbor joining algorithm and general time reversible for the nucleotide substitution model. Branching values were determined using

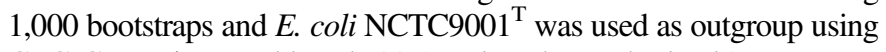
CLC Genomics Workbench 11 (Venlo, The Netherlands).

\section{RESULTS}

There were significant differences in bacterial composition depending on the health status of the mushrooms $(P=0.001)$ and sampling method $(P=0.009)$ (Fig. 2A). Within the mushroom cap, Proteobacteria and Bacteroidetes were the most abundant bacterial phyla regardless of extraction method or mushroom health status (Fig. 2B). No significant differences were detected in the interaction between health status and sampling method $(P=0.929)$ (Fig. 2A). Thus, we discuss the main effects (sampling method or health status) in separate sections below.

With regards to alpha diversity, no significant interactions were observed between sampling method and mushroom health based on Shannon diversity $(P=0.7320)$, OTU richness $(P=0.7940)$, and Pielou's evenness $(P=0.7498)$. Among the three bacterial sampling methods, washate and post-washate exhibited the highest Shannon diversity $(P=0.027)$ and Pielou's evenness $(P=0.0359)$ (Fig. $3 \mathrm{AC})$. However, no significant differences were detected for observed OTU richness $(P=0.312)$ (Fig. 3B). When we compared diversity metrics based on mushroom health status, we observed higher Shannon diversity $(P<0.001)$, OTU richness $(P<0.001)$, and Pielou's evenness $(P=0.004)$ in asymptomatic mushrooms (Fig. 3A to C).
A NMDS of global 16S rRNA gene composition

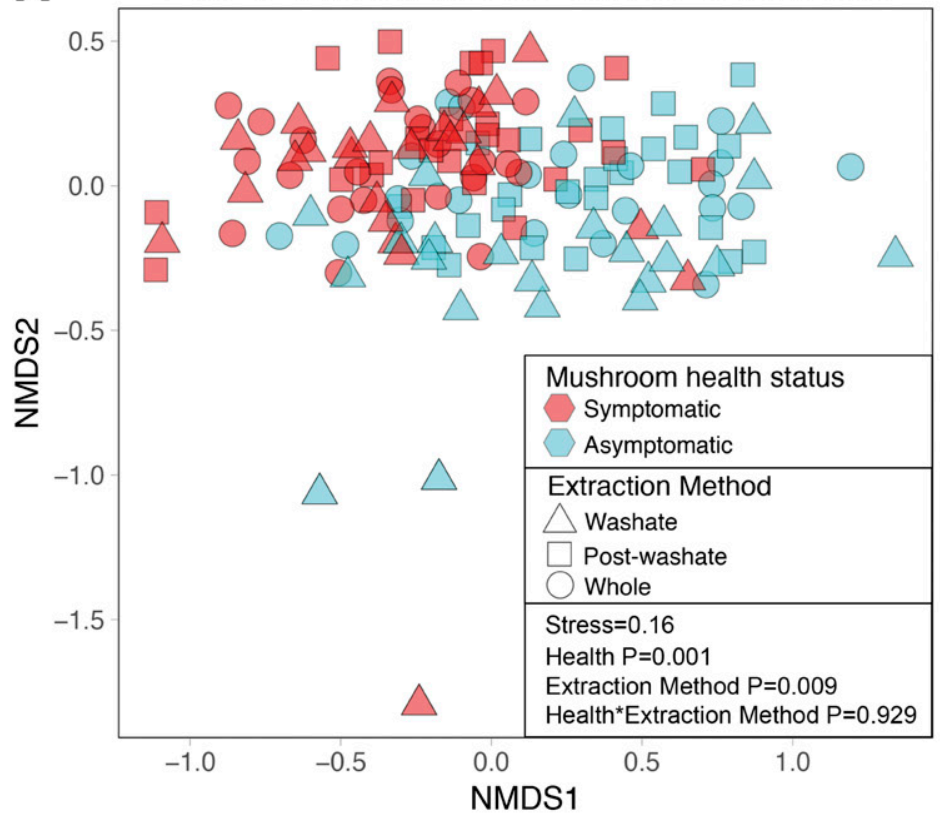

B

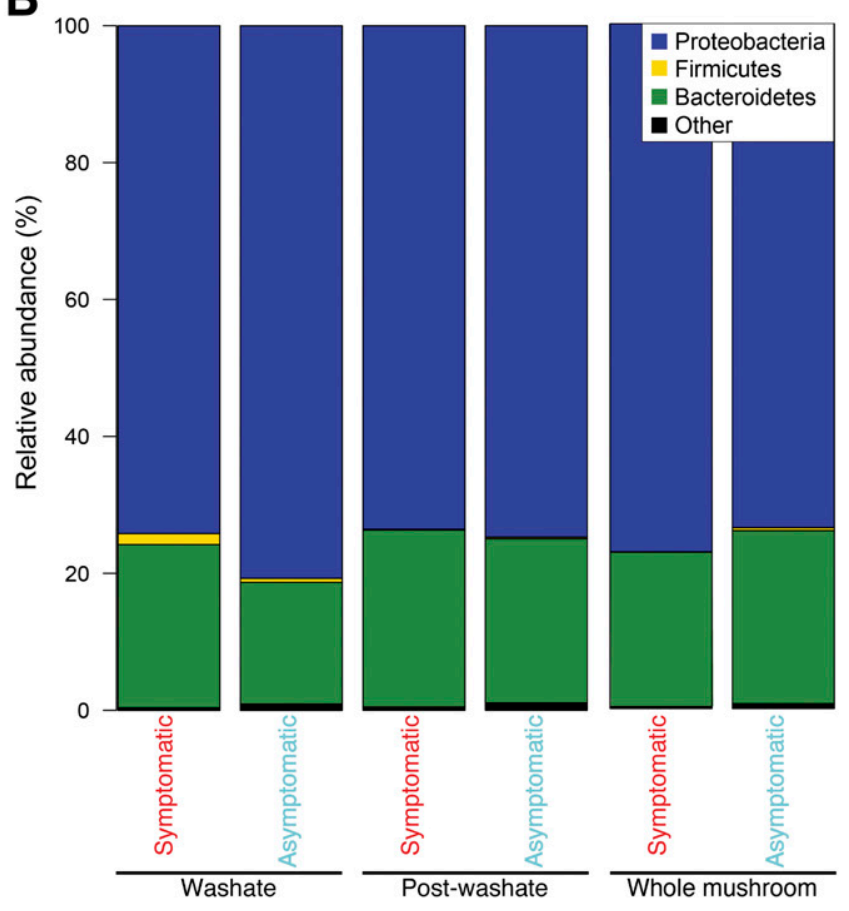

Fig. 2. A, Nonmetric multidimensional scaling (NMDS) of global 16S rRNA gene composition from symptomatic and asymptomatic Agaricus bisporus extracted through different methods (washate, post-washate, and whole). B, Relative abundance of operational taxonomic units at the phylum level. 
Overall difference between asymptomatic and symptomatic mushroom microbiome. As stated previously, differences were observed in bacterial composition depending on the health status of the mushroom cap $(P=0.001)$. The relative abundance of most bacterial families was comparable in both symptomatic and asymptomatic mushrooms (Fig. 4, branches and nodes in gray). However, several families were more abundant in asymptomatic mushrooms (branches and nodes colored in cyan). In contrast, only a few families, including Enterobacteriaceae, Sphingobacteriaceae, Flavobacteriaceae, and Weeksellaceae, were more abundant in or on symptomatic mushrooms (branches and nodes colored in red).

Comparison between the methods (washate and post-washate mushroom) and mushroom health status. We compared washate and post-washate bacterial composition from the same individual mushroom caps because these samples together should encompass the entire mushroom cap microbiome, and because they potentially represent two distinct components of the microbiome: bacteria that are easily removed from the cap by sonication (washate) and bacteria that remain associated with the mushroom after sonication and are potentially internal to the cap (post-washate). For both symptomatic and asymptomatic mushrooms, most taxa were equally abundant in both microbiome components (Fig. 5A branches and nodes in gray). However, there were more significantly overrepresented taxa in the tightly adhering/internal component (post-washate, shown in green) than in the component that is easily removed by sonication (washate), regardless of health status. For instance, there were 14 and 36 more overrepresented taxa tightly adhering to the mushrooms for symptomatic (Fig. 5B) and asymptomatic (Fig. 5C) mushrooms, respectively. Among the Proteobacteria, the most abundant phylum for all treatments (Fig. 2B), the Gammaproteobacteria were overrepresented in the lightly adhering component (washate, $P>0.05$ ), while Alphaproteobacteria were overrepresented in the tightly adhering/internal component (post-washate, $P>0.001$ ) (Fig. 5C).

Interestingly, particular taxa differed with respect to whether they were overrepresented in one of the microbiome components depending on mushroom health status. For instance, regardless of visible mushroom health status, the phylum Acidobacteria was more abundant in the lightly adhering component represented by the washate method (shown in brown). In contrast, the phyla Armatimonadetes and Gemmatimonadetes as well as the classes Spartobacteria, Pedosphaerae, and Opitutae from the phylum Verrucomicrobia were only overrepresented in the tightly adhering/ internal component (post-washate) and only from asymptomatic mushrooms (Fig. 5B and C). Likewise, the families Chthoniobacteraceae and Opitutaceae were also overrepresented in asymptomatic mushrooms but only in the lightly adhering component (washate, Fig. 5C, shown in brown).

Globally, the genus Pseudomonas was overrepresented by the washate method (Fig. 5D). Because of this and the importance of the genus to mushroom development and health, we further analyzed the exact sequence variances (ESVs) from the genus Pseudomonas. Most of the ESVs were distributed evenly among all of the samples, regardless of health status or microbiome component (Fig. 6). Four ESVs (ESV565, ESV1109, ESV221, and ESV1008) were most closely related to the mushroom pathogen $P$. agarici (Fig. 6). This was not the case for all blotch pathogens however. For example, strains isolated from Pennsylvania mushroom houses and previously identified as P. tolaasii (S. Martins et al., unpublished data; BP1104, BP1106, BP1199, BP1210, BP1243, BP1271, and BP1242) were all within a large clade that included the type strain of $P$. tolaasii and several other type strains of fluorescent pseudomonads. There were two ESVs in this clade, but we cannot confirm whether or not they truly represent $P$. tolaasii strains in these samples. Sequences for other unnamed Pennsylvania blotch pathogens (S. Martins et al., unpublished data) were nearly identical to some of the ESVs detected (e.g., BP1286 and ESV31).

\section{DISCUSSION}

The long-term goal of our research is to understand the microbial factors influencing spatiotemporal dynamics of bacterial blotch pathogens from the genus Pseudomonas on mushroom (A. bisporus) caps and disease development with the goal of manipulating these pathogens to increase commercial mushroom yield and quality. Results from a variety of studies using culture-dependent methods lead researchers to hypothesize that the mushroom cap
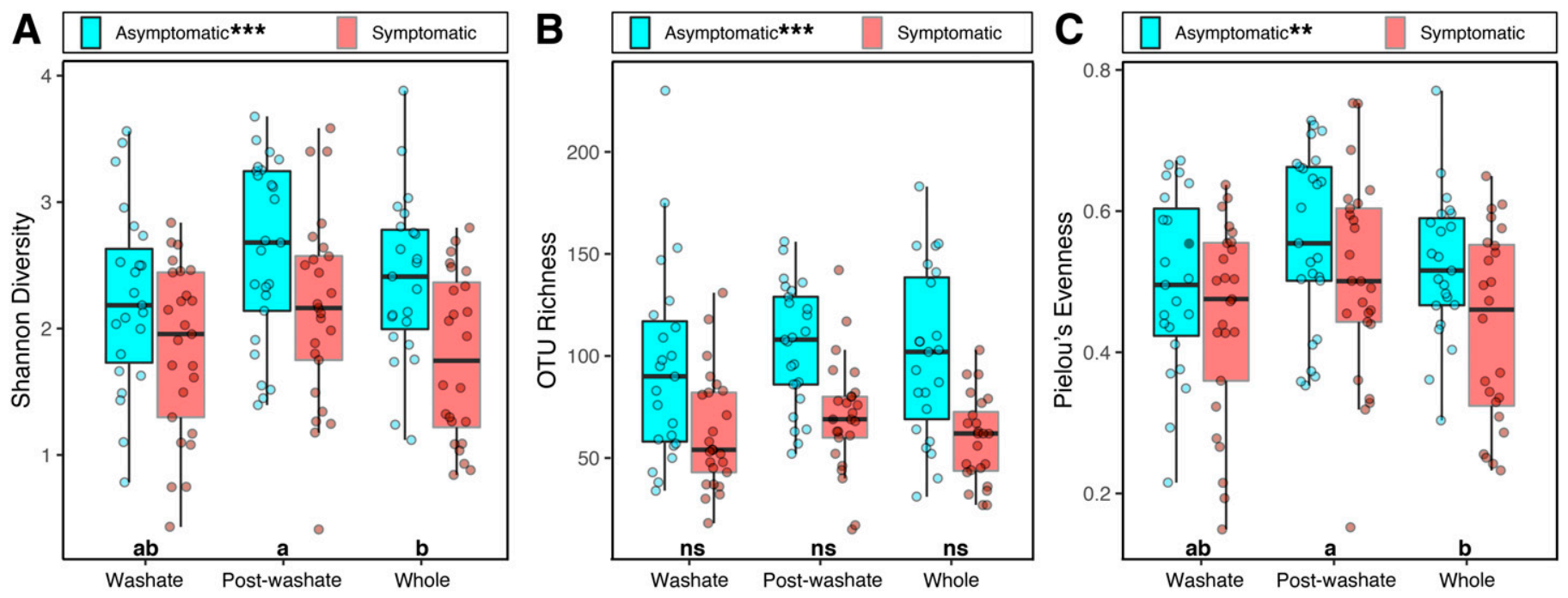

Fig. 3. Comparison among bacterial isolation methods (washate, post-washate, and whole) and between mushroom (Agaricus bisporus) health status (asymptomatic and symptomatic). A, Shannon diversity; B, operational taxonomic unit (OTU) richness; and C, Pielou's evenness. Among the methods, bars with the same lowercase letter are similar at the $5 \%$ level according to Tukey's test. Between mushroom health, ${ }^{* *}$ indicates significant at the 0.01 probability level; and ${ }^{* * *}$ indicates significant at the 0.001 probability level. $n s=$ not significant. 
microbiome influences mushroom pathogen composition and survival and resulting disease development (Nguyen et al. 2012; Saxon et al. 2014). Technical advances now allow description of mushroom cap microbiomes and bacterial succession on cultivated and wild mushrooms using culture-independent techniques (Pent et al. 2018; Siyoum et al. 2016; Xia et al. 2019). The data presented here provide several significant advances for our work and research in this field. This is the first research using culture-independent methods to demonstrate that distinct bacterial communities are present on mushrooms caps whose health status can be differentiated as either symptomatic or asymptomatic through visual observation. Next, the novel finding that the sampling approach significantly impacts the description of microbiomes associated with mushroom caps (Figs. 2A, 3A and C, and 5) may indicate that the tightly adhering/internal bacteria are compositionally distinct from those that are loosely associated with the mushroom cap. This research provides a preliminary evaluation of an ESV diagnostic approach for describing pathogen communities and demonstrated its value and limitations for identifying various mushroom blotch pathogens.
Culture-dependent studies on mushroom cap health, and bacterial blotch in particular, have demonstrated the potential influence of one microbe or a set of microbes on pathogen populations on cultivated mushroom caps. For example, Bdellovibrio bacteriovorus is known to reduce the abundance of individual pathogens on the surface of mushrooms and reduce blotch on postharvest conditions (Saxon et al. 2014). Likewise, a novel bacteriophage, $\varphi$ Pto-bp6g, was found to lyse P. tolaasii and control brown blotch disease in mushrooms (Pleurotus ostreatus) (Nguyen et al. 2012). Furthermore, there is preliminary data that indicate that blotch pathogens produce compounds that inhibit each other and other Pseudomonas species (R. Hershlag et al., unpublished data) and additional examples were recently reviewed (Osdaghi et al. 2019). However, in vitro and single strain pairings can only describe the potential of an organism to be involved in shaping microbial communities or in microbial community dynamics. In situ studies of bacterial communities are needed to understand the complex web of relationships in the environment where they take place.

Fungal, yeast, and bacterial composition all changed over the mushroom cropping cycle (Siyoum et al. 2016). Because the

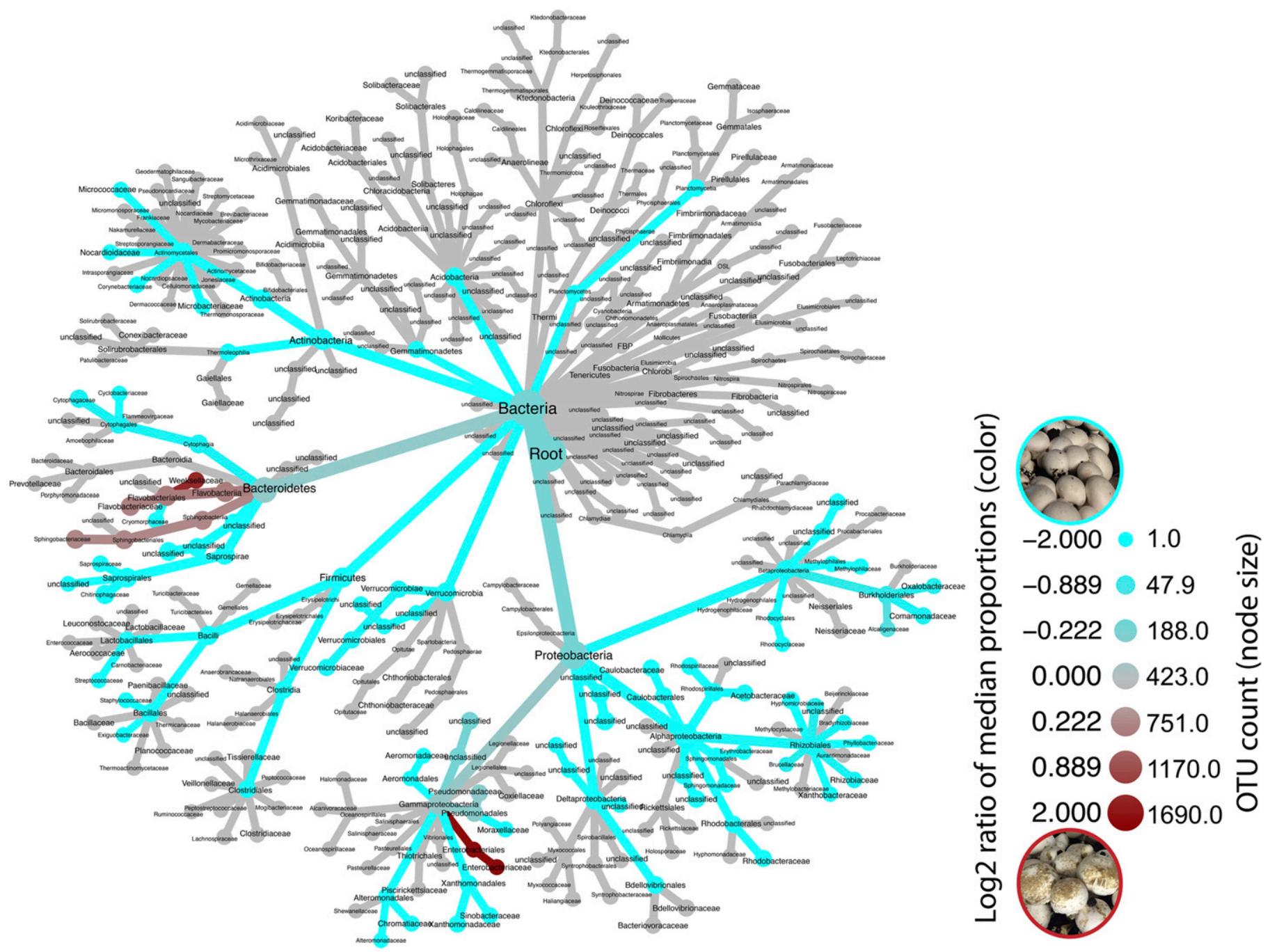

Fig. 4. Differential heat tree showing relative abundance of bacteria among all the taxa, up to the family level between asymptomatic (cyan) and symptomatic (red) mushrooms (Agaricus bisporus). The data presented are a combination of all the bacterial isolation methods (washate, postwashate, and whole). For each taxon, a Wilcoxon rank sum test was used to assess differences between the median abundances of samples in each treatment (mushroom health status). The branches indicate the association between taxa and the node sizes indicate the operational taxonomic unit (OTU) counts per taxon. Taxa in red are more abundant in symptomatic mushrooms, while those in cyan are more abundant in asymptomatic mushrooms. Taxa in gray were equally detected in both symptomatic and asymptomatic mushrooms. 
authors found that bacteria were the more abundant compared with yeast and fungi, this study concentrated exclusively on bacterial composition. In order to understand what influence bacterial succession, and in particular pathogen succession is having on mushroom health status, it was necessary to first determine whether there is a difference in microbiome composition on mushroom caps that are visibly observable as either symptomatic or asymptomatic. A second reason for concentrating on bacteria was that it helps to characterize potential relationships between blotch pathogens and interactions with other bacteria.

The data presented here demonstrate that bacterial composition differed between symptomatic and asymptomatic mushrooms (Fig. 2A), with higher bacterial diversity observed in asymptomatic rather than symptomatic mushrooms (Fig. 3A). For example, there were 14 and 36 more overrepresented taxa internal or tightly adhering to the mushrooms for symptomatic (Fig. 5B) and asymptomatic (Fig. 5C) mushrooms, respectively. Particular families within the phyla Planctomycetes, Acidobacteria, Gammatimonadetes, Actinobacteria, Firmicutes, and Verrucomicrobia were responsible for this overrepresentation (Fig. 4). Barring artifacts due to overrepresentation of individual taxa in the symptomatic mushrooms, the overrepresented taxa from asymptomatic mushroom caps will serve as targets for future research to determine whether they are involved in inhibiting blotch pathogens and reducing the incidence or severity of disease on mushroom caps.

Alternatively, the significantly lower diversity (Fig. 3A), richness (Fig. 3B), and evenness (Fig. 3C) of bacteria in symptomatic compared with asymptomatic mushrooms might be explained by suppression of other bacteria by the dominant pathogens (e.g.,
Pseudomonas) associated with symptomatic mushrooms. For instance, Pseudomonas spp. produce active proteinases and lipases, which increase fitness against other microorganisms in the $A$. bisporus system (Chung et al. 2014). Likewise, P. tolaasii produces volatile organic compounds (Lo Cantore et al. 2015), which could also inhibit the growth of other microbes in symptomatic mushrooms, due to the broad spectrum of these compounds (Martins et al. 2019; Terra et al. 2018). We have preliminary data that indicate that blotch pathogens produce compounds that inhibit each other and other Pseudomonas species (R. Hershlag et al., unpublished data). Antibiosis by Pseudomonas spp. may also be responsible for the underrepresentation of certain taxa (e.g., Planctomycetes, Acidobacteria, Gammatimonadetes, Actinobacteria, Firmicutes, and Verrucomicrobia) on symptomatic mushrooms.

Some taxa, such as the families Enterobacteriaceae and Sphingobacteriaceae, were more abundant in symptomatic mushrooms (Supplementary Table S1). It is not known whether these organisms are overabundant because they are not inhibited by Pseudomonas spp. and can colonize at higher densities due to the lack of competitors. Alternatively, the correlated organisms may interact synergistically with blotch pathogens. For example, members of family Sphingobacteriaceae produce catalase and oxidase (Lambiase 2014), which may be involved in cell wall degradation in mushroom. However, there is no published link between the presence of bacteria from the families Enterobacteriaceae and Sphingobacteriaceae and blotch in mushroom.

The second significant advance from this work is the finding that sampling approach can impact the resulting description of the

Washate mushroom VS Post-washate mushroom

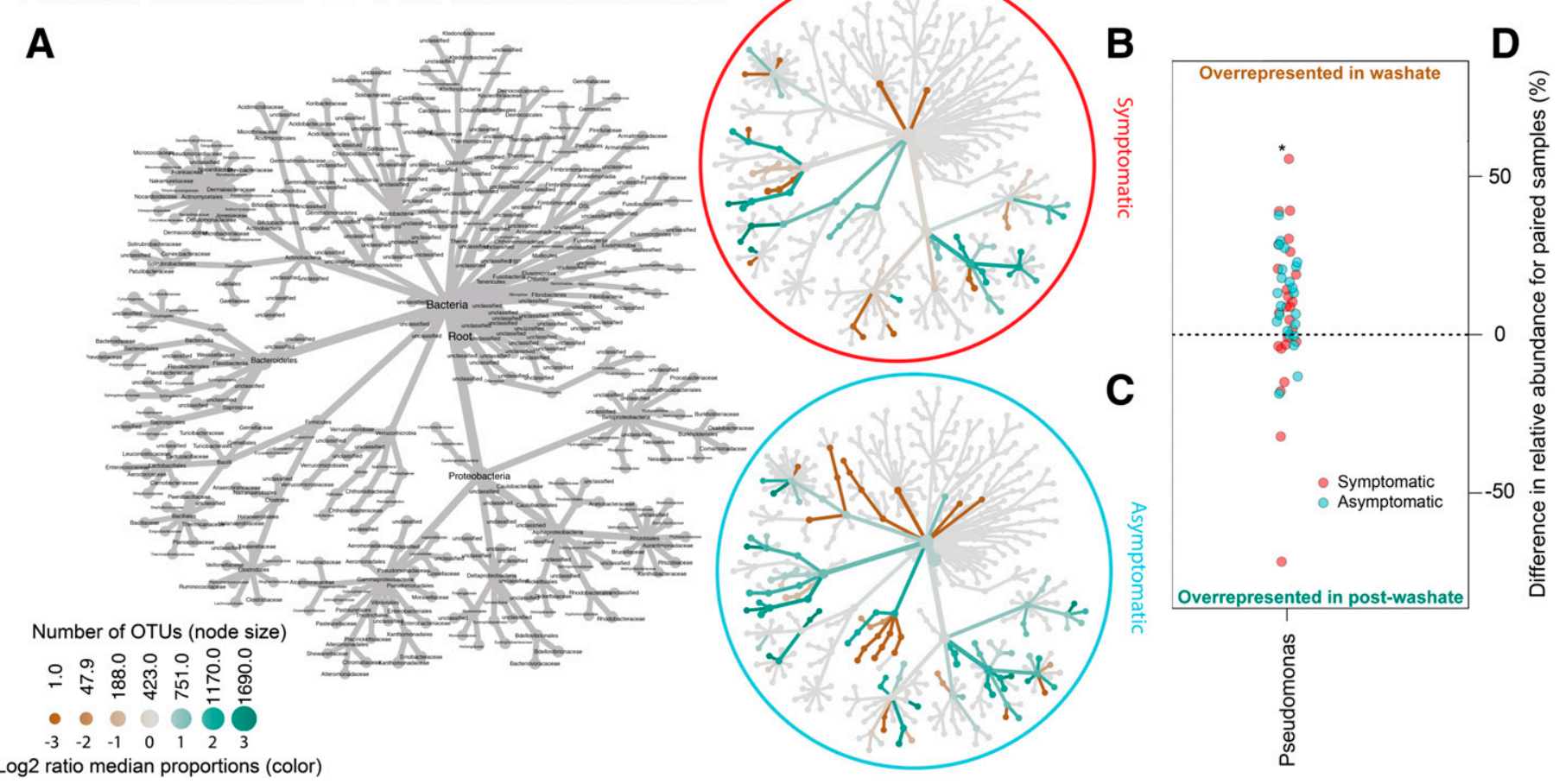

Fig. 5. Differential heat tree showing differences in bacterial composition to the family level. A, The gray tree on the left functions as a key for the smaller unlabeled trees. The comparisons were made among the methods of bacterial isolation post-washate and washate from $\mathbf{B}$, symptomatic and $\mathbf{C}$, asymptomatic mushrooms (Agaricus bisporus). For each taxon, a Wilcoxon rank sum test was used to test for differences between the median abundances of samples in each treatment. Taxa in brown are statistically more abundant in the washate method, while those in green are statistically more abundant in the post-washate method. The branches indicate the association between taxa and the node sizes indicate the operational taxonomic unit (OTU) counts per taxon. D, Comparison between washate and post-washate methods and resolving asymptomatic and symptomatic for Pseudomonas spp. *Significant at the 0.05 probability level by Student's $t$ test. 
bacterial composition associated with mushrooms caps (Figs. 2A, $3 \mathrm{~A}$ and $\mathrm{C}$, and 5). Compared with the washate/post-washate methods, the whole mushroom did not differ regarding the two most abundant phyla, Proteobacteria and Bacteriodetes (Fig. 2B). However, a lower Shannon diversity and Pielou's evenness were found for the bacteria present in samples when the whole mushrooms were sampled compared with the samples where the loosely or tightly adhering bacteria were analyzed separately (Fig. 3). The combining of the loosely or tightly adhering bacterial fractions in the whole microbiome samples appears to quench the signals from less abundant bacterial sequences in either fraction because both Shannon diversity and Pielou's evenness respond to abundance. These sampling protocols can now be used to strategically target given populations. For example, more Pseudomonas spp. were found in the washate of symptomatic and asymptomatic mushrooms. This diversity largely represents the overall Pseudomonas

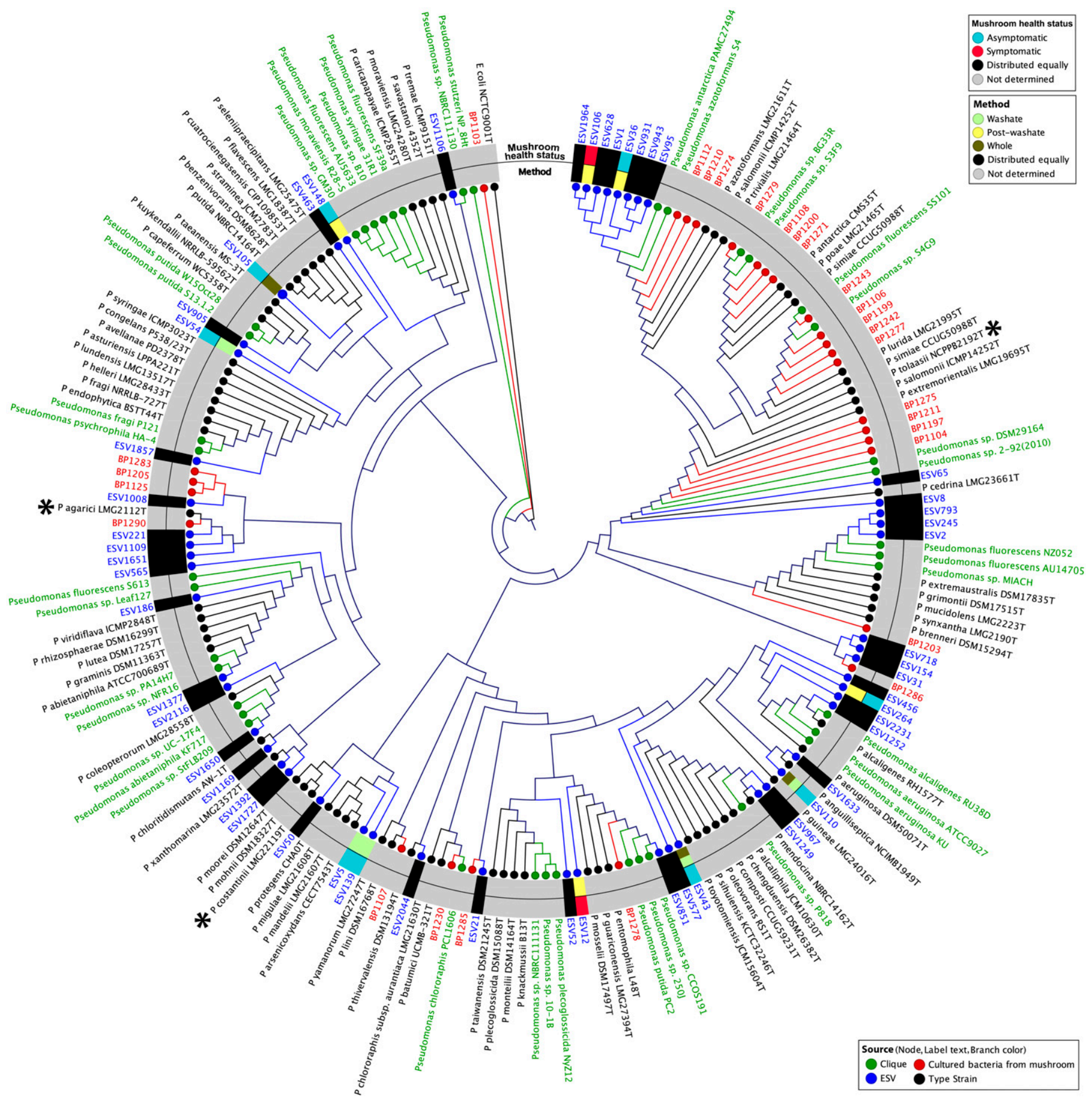

Fig. 6. Comparisons of exact sequence variants (ESVs) to type and reference strains from known Pseudomonas spp. The node, text, and branch of 27 cultured blotch-causing bacteria isolated from mushroom (Agaricus bisporus), Pseudomonas type strains, and the ESVs are labeled, respectively, in red, blue, and green. The asterisks $\left(^{*}\right)$ before the bacterial names indicate type strains of known blotch-causing agents. Neighbor joining algorithm and general time reversible were used for the nucleotide substitution model. Branching values were determined using 1,000 bootstraps. Escherichia coli NCTC9001T was used as outgroup. 
composition of the mushroom cap and could be used as a surrogate for the whole cap. Additionally, pathogenic Pseudomonas species causing blotch colonize the mushroom surface tissues prior to initiating disease (Saxon et al. 2014). Alternatively, it may be strategic to use post-washate samples to study or isolate bacteria other than pseudomonads for biological control because of their association with asymptomatic mushrooms. For example, families in Bacillales, Bdellovibrionales, and Xanthomonadales were among the 33 taxa that were more abundant in the internal or tightly adhering component of asymptomatic mushroom microbiomes, and members of these families have been reported to provide biocontrol responses against blotch in mushroom (Aslani et al. 2018; Saxon et al. 2014; Tajalipour et al. 2014).

Bacteria in the Proteobacteria and Bacteroidetes are a dominant feature in mushroom production. Like other research on $A$. bisporus (Godfrey et al. 2001; Siyoum et al. 2016; Yang et al. 2019) and wild mushroom (Venturini et al. 2011) cap microbiomes, we observed that mushroom cap microbiomes were predominantly composed of sequences from members of Proteobacteria and Bacteroidetes (Fig. 2B). This work demonstrated for the first time that Proteobacteria and Bacteroidetes were consistently distributed regardless of bacterial isolation method or mushroom health status (Fig. 2B). It is hypothesized that the caps are colonized with bacteria from the growth substrate. For example, a peat moss-based casing layer showed a higher relative abundance of Proteobacteria and Bacteroidetes (Pecchia et al. 2014). However, the dominant bacteria in the compost differ from those found in the mushroom caps and casing layer. Firmicutes comprise the majority of bacteria prior to the introduction of $A$. bisporus inoculum to compost (Vieira and Pecchia 2018).

The bacterial profiles of $A$. bisporus mushroom caps were primarily composed of Pseudomonas spp. $(\sim 60 \%$ of the total sequences in this study), i.e., members of Proteobacteria (Fig. 2B), which is in accordance with other studies (Godfrey et al. 2001; Siyoum et al. 2016; Yang et al. 2019). In developing strategies for microbiome manipulation and reducing pathogen populations, it is important to remain cognizant that members of the genus Pseudomonas are important members of the mushroom devome (organisms and outcomes of microbial mediated development). Thus, adequate sequence-based taxonomic or pathogen-specific markers and techniques are needed to differentiate pathogenic from beneficial Pseudomonas spp. or strains. The research reported here coupled to culture-dependent methods, which circumscribed the diversity of pathogens present in Pennsylvania mushroom farms (S, Martins et al., unpublished data) and sequencing of type strains of 166 species or subspecies and identification of reference strains for an additional 189 unnamed species of Pseudomonas, serves as the first proof of concept for ESV diagnostics for bacterial blotch of mushrooms or other diseases. The data indicate that this method was predictive for previously identified $P$. agarici strains. Six ESVs were most closely related to the same region in the mushroom pathogen $P$. agarici and other blotch pathogens from Pennsylvania. Additionally, sequences for other unnamed Pennsylvania blotch pathogens were similar to detected ESVs (e.g., BP1286 and ESV21). Although, isolation of strains with these ESVs from these samples followed by testing of pathogens from these communities would provide final confirmation of the presence of these pathogens in the samples with these ESVs, it appears that ESVs from this 16S rRNA gene fragment may be diagnostic for some pathogens.

Previously, $P$. protegens, a soilborne organism used for biological control of soilborne fungal pathogens of plants, was shown to cause mushroom browning and discoloration similar to symptoms produced by blotch pathogens (Henkels et al. 2014). To our knowledge, it has never been isolated from mushrooms with blotch from a commercial mushroom facility or elsewhere. The data presented here indicate that $P$. protegens was not present in or on the mushrooms sampled as none of the ESVs were closely related to $P$. protegens.

It is likely that the ESV approach with this sequence fragment is not diagnostic for the most important blotch pathogen, $P$. tolaasii. The DNA sequences of this fragment for previously identified $P$. tolaasii strains (S. Martins et al., unpublished data) encompass 14 different named species including $P$. tolaasii. Some ESVs from the samples evaluated in this research were grouped close to the pathogens identified in our previous study, but it is not clear whether they are likely to represent $P$. tolaasii or other Pseudomonas spp. Other genes and sequence fragments are being explored that are likely to provide better resolution for $P$. tolaasii and other blotchcausing Pseudomonas spp. (Aleman et al. 2019).

This research made strides in describing the mushroom cap microbiome and established sampling methods that will allow different segments of the bacterial community to be targeted including pathogens in the genus Pseudomonas and organisms with potential for biological control. Although the ESV diagnostic approach may be sufficient for some pathogenic species (P. agarici), it is clear from this work that sequences from fragments of other genes will be needed to detect the most important bacterial blotch pathogen (P. tolaasii) from mushroom and environmental samples.

\section{ACKNOWLEDGMENTS}

A preliminary report of this research was made at the 2018 Penn State Wild and Tamed Phytobiomes Conference sponsored by the Phytobiomes Journal. Assistance in sample processing and initial data exploration was provided by Tarik Acevedo, Amanda Mainello, Megan Campbell, Siyi Ge, Sofia Roitman, and June Teichmann. Special thanks to the Penn State Microbiome Center (@PSUmBiome) and the Department of Plant Pathology and Environmental Microbiology (@psuPPEM) for additional support.

\section{LITERATURE CITED}

Aleman, M., Bull, C. T., and Hockett, K. L. 2019. Understanding microbial communities: Single-gene phylogenies and their resolution. Plant Health 2019, Cleveland, OH.

Apprill, A., McNally, S., Parsons, R., and Weber, L. 2015. Minor revision to V4 region SSU rRNA 806R gene primer greatly increases detection of SAR11 bacterioplankton. Aquat. Microbiol. Ecol. 75:129-137.

Aslani, M. A., Harighi, B., and Abdollahzadeh, J. 2018. Screening of endofungal bacteria isolated from wild growing mushrooms as potential biological control agents against brown blotch and internal stipe necrosis diseases of Agaricus bisporus. Biol. Control 119:20-26.

Callahan, B. J., McMurdie, P. J., Rosen, M. J., Han, A. W., Johnson, A. J., and Holmes, S. P. 2016. DADA2: High-resolution sample inference from Illumina amplicon data. Nat. Methods 13:581-583.

Caporaso, J. G., Kuczynski, J., Stombaugh, J., Bittinger, K., Bushman, F. D., Costello, E. K., Fierer, N., Gonzalez Pena, A., Goodrich, J. K., Gordon, J. I., Huttley, G. A., Kelley, S. T., Knights, D., Koenig, J. E., Ley, R. E., Lozupone, C. A., McDonald, D., Muegge, B. D., Pirrung, M., Reeder, J., Sevinsky, J. R., Turnbaugh, P. J., Walters, W. A., Widmann, J., Yatsunenko, T., Zaneveld, J., and Knight, R. 2010. QIIME allows analysis of high-throughput community sequencing data. Nat. Methods 7:335-336.

Chang, S. T., and Wasser, S. P. 2017. Page 43 in: The Cultivation and Environmental Impact of Mushrooms. Printed from the Oxford Research Encyclopedia, Environmental Science. Oxford University Press.

Chung, I. Y., Kim, Y. K., and Cho, Y. H. 2014. Common virulence factors for Pseudomonas tolaasii pathogenesis in Agaricus and Arabidopsis. Res. Microbiol. 165:102-109.

Edgar, R. C. 2010. Search and clustering orders of magnitude faster than BLAST. Bioinformatics 26:2460-2461.

Foster, Z. S. L., Sharpton, T., and Grunwald, N. J. 2016. MetacodeR: An R package for visualization and manipulation of community taxonomic diversity data. PLoS Comput. Biol. 13:e1005404. 
Godfrey, S. A. C., Harrow, S. A., Marshall, J. W., and Klena, J. D. 2001. Characterization by $16 \mathrm{~S}$ rRNA sequence analysis of pseudomonads causing blotch disease of cultivated Agaricus bisporus. Appl. Environ. Microbiol. 67: 4316-4323.

Henkels, M. D., Kidarsa, T. A., Shaffer, B. T., Goebel, N. C., Burlinson, P., Mavrodi, D. V., Bentley, M. A., Rangel, L. I., Davis, E. W., II, Tomashow, L. S., Zabriskie, T. M., Preston, G. M., and Loper, J. E. 2014. Pseudomonas protegens $\mathrm{Pf}-5$ causes discoloration and pitting of mushroom caps due to the production of antifungal metabolites. Mol. Plant-Microbe Interact. 27: 733-746.

Hesse, C., Schulz, F., Bull, C. T., Shaffer, B. T., Yan, Q., Shapiro, N., Hassan, K. A., Varghese, N., Elbourne, L. D. H., Paulsen, I. T., Kyrpides, N., Woyke, T., and Loper, J. E. 2018. Genome-based evolutionary history of Pseudomonas spp. Environ. Microbiol. 20:2142-2159.

Howard, M. M., Bell, T. H., and Kao-Kniffin, J. 2017. Soil microbiome transfer method affects microbiome composition, including dominant microorganisms, in a novel environment. FEMS Microbiol. Lett. 364:fnx092.

Kertesz, M. A., and Thai, M. 2018. Compost bacteria and fungi that influence growth and development of Agaricus bisporus and other commercial mushrooms. Appl. Microbiol. Biotechnol. 102:1639-1650.

Kozich, J. J., Westcott, S. L., Baxter, N. T., Highlander, S. K., and Schloss, P. D. 2013. Development of a dual-index sequencing strategy and curation pipeline for analyzing amplicon sequence data on the MiSeq Illumina sequencing platform. Appl. Environ. Microbiol. 79:5112-5120.

Lambiase, A. 2014. The family Sphingobacteriaceae. Pages 907-9014 in: The Prokaryotes, 4th Ed. E. Rosenberg, E. F. DeLong, S. Lory, E. Stackebrandt, F. Thompson, et al., eds. Springer, Heidelberg, Germany.

Lo Cantore, P., Giorgio, A., and Iacobellis, N. S. 2015. Bioactivity of volatile organic compounds produced by Pseudomonas tolaasii. Front. Microbiol. 6: 1082.

Love, M. I., Huber, W., and Anders, S. 2014. Moderated estimation of fold change and dispersion for RNA-seq data with DESeq2. Genome Biol. 15:550.

Martinez-Carrera, D., Sobal, M., Aguilar, A., Navarro, M., Bonilla, M., and Larque-Saavedra, A. 1998. Canning technology as an alternative for management and conservation of wild edible mushrooms in Mexico. Micol. Neotrop. Apl. 11:35-51.

Martins, S. J., Faria, A. F., Medeiros, F. H. V., Pedroso, M. P., Cunha, M. G., and Rocha, M. R. 2019. Microbial volatiles organic compounds control anthracnose (Colletotrichum lindemuthianum) in common bean (Phaseolus vulgaris L.). Biol. Control 131:36-42.

McDonald, D., Price, M. N., Goodrich, J., Nawrocki, E. P., DeSantis, T. Z., Probst, A., Andersen, G. L., Knight, R., and Hugenholtz, P. 2012. An improved Greengenes taxonomy with explicit ranks for ecological and evolutionary analyses of bacteria and archaea. ISME J. 6:610-618.

McMurdie, P. J., and Holmes, S. 2013. phyloseq: An R package for reproducible interactive analysis and graphics of microbiome census data. PLoS One 8: e61217.

McMurdie, P. J., and Holmes, S. 2014. Waste not, want not: Why rarefying microbiome data is inadmissible. PLoS Comput. Biol. 10:1003531.

Munsch, P., Alatossava, T., Marttinen, N., Meyer, J. M., Christen, R., and Gardan, L. 2002. Pseudomonas costantinii sp. nov., another causal agent of brown blotch disease, isolated from cultivated mushroom sporophores in Finland. Int. J. Syst. Evol. Microbiol. 52:1973-1983.

Navarro, M. J., Geaa, F. J., and González, A. J. 2018. Identification, incidence and control of bacterial blotch disease in mushroom crops by management of environmental conditions. Sci. Hortic. (Amsterdam) 229:10-18.

Nguyen, H. T. D., Yoon, S., Kim, M. K., Kim, Y. K., Yoon, M. Y., Cho, Y. H., Lim, Y., Shin, S. H., and Kim, D. E. 2012. Characterization of bacteriophage $\varphi$ Pto-bp6g, a novel phage that lyses Pseudomonas tolaasii causing brown blotch disease in mushrooms. J. Microbiol. Methods 91:514-519.

Noble, R., Fermor, T. R., Lincoln, S., Dobrovin-Pennington, A., Evered, C., Mead, A., and Li, R. 2003. Primordia initiation of mushroom (Agaricus bisporus) strains on axenic casing materials. Mycologia 95:620-629.

Oksanen, J., Kindt, R., Legendre, P., O’Hara, B., Simpson, G. L., Solymos, P., Stevens, M. H. H., and Wagner, H. 2015. Vegan community ecology package. http://vegan.r-forge.r-project.org/

Osdaghi, E., Martins, S. J., Sepulveda, L., Vieira, F. R., Pecchia, J. A., Beyer, D. M., Bell, T. H., Yang, Y., Hockett, K. L., and Bull, C. T. 2019. 100 years since Tolaas: Bacterial blotch of mushrooms in the 21st century. Plant Dis. 103: 2714-2732.
Paine, S. G. 1919. A brown blotch disease of cultivated mushrooms. Ann. Appl. Biol. 5:206-219.

Parada, A. E., Needham, D. M., and Fuhrman, J. A. 2016. Every base matters: Assessing small subunit rRNA primers for marine microbiomes with mock communities, time series and global field samples. Environ. Microbiol. 18: 1403-1414.

Pecchia, J., Cortese, R., and Albert, I. 2014. Investigation into the microbial community changes that occur in the casing layer during cropping of the white button mushroom, Agaricus bisporus. Pages 309-313 in: Proceedings of the 8th International Conference on Mushroom Biology and Mushroom Products. M. Singh, ed. New Delhi, India.

Pent, M., Hiltunen, M., Põldmaa, K., Furneaux, B., Hildebrand, F., Johannesson, H., and Bahram, M. 2018. Host genetic variation strongly influences the microbiome structure and function in fungal fruiting-bodies. Environ. Microbiol. 20:1641-1650.

Pylro, V. S., Roesch, L. F., Ortega, J. M., Amaral, A. M., Tótola, M. R., Hirsch, P. R., Rosado, A. S., Góes-Neto, A., da Costa da Silva, A. L., Rosa, C. A., Morais, D. K., Andreote, F. D., Duarte, G. F., de Melo, I. S., Seldin, L., Lambais, M. R., Hungria, M., Peixoto, R. S., Kruger, R. H., Tsai, S. M., and Azevedo, V. 2014. Brazilian microbiome project: Revealing the unexplored microbial diversity - Challenges and prospects. Microbiol. Ecol. 67:237-241.

Quast, C., Pruesse, E., Yilmaz, P., Gerken, J., Schweer, T., Yarza, P., Peplies, J., and Glöckner, F. O. 2012. The SILVA ribosomal RNA gene database project: Improved data processing and web-based tools. Nucleic Acids Res. 41: D590-D596.

Rossouw, W., and Korsten, L. 2017. Cultivable microbiome of fresh white button mushrooms. Lett. Appl. Microbiol. 64:164-170.

Royse, D. J., Baars, J., and Tan, Q. 2017. Current overview of mushroom production in the world. Pages 5-13 in: Edible and Medicinal Mushrooms: Technology and Applications. D. C. Zied and A. Pardo-Giménez, eds. John Wiley \& Sons Ltd., Chichester, Sussex.

Saxon, E. B., Jackson, R. W., Bhumbra, S., Smith, T., and Sockett, R. E. 2014. Bdellovibrio bacteriovorus HD100 guards against Pseudomonas tolaasii brown-blotch lesions on the surface of post-harvest Agaricus bisporus supermarket mushrooms. BMC Microbiol. 14:163.

Schloss, P. D., Westcott, S. L., Ryabin, T., Hall, J. R., Hartmann, M., Hollister, E. B., Lesniewski, R. A., Oakley, B. B., Parks, D. H., Robinson, C. J., Sahl, J. W., Stres, B., Thallinger, G. G., Horn, D. J. V., and Weber, C. F. 2009. Introducing mothur: Open-source, platform-independent, communitysupported software for describing and comparing microbial communities. Appl. Environ. Microbiol. 75:7537-7541.

Siyoum, N. A., Surridge, K., van der Linde, E. J., and Korsten, L. 2016. Microbial succession in white button mushroom production systems from compost and casing to a marketable packed product. Ann. Microbiol. 66: 151-164.

Tajalipour, S., Hassanzadeh, N., Jolfaee, H. K., Heydari, A., and Ghasemi, A. 2014. Biological control of mushroom brown blotch disease using antagonistic bacteria. Biocontrol Sci. Technol. 24:473-484.

Terra, W. C., Campos, V. P., Martins, S. J., Costa, L. S. A. S., da Silva, J. C. P., Barros, A. F., Lopez, L. E., Santos, T. C. N., and Smant, G. 2018. Volatile organic molecules from Fusarium oxysporum strain 21 with nematicidal activity against Meloidogyne incognita. Crop Prot. 106:125-131.

Tolaas, A. G. 1915. A bacterial disease of cultivated mushrooms. Phytopathology 5:51-54.

Venturini, M. E., Reyes, J. E., Rivera, C. S., Oria, R., and Blanco, D. 2011. Microbiological quality and safety of fresh cultivated and wild mushrooms commercialized in Spain. Food Microbiol. 28:1492-1498.

Vieira, F. R., and Pecchia, J. A. 2018. An exploration into the bacterial community under different pasteurization conditions during substrate preparation (composting-phase II) for Agaricus bisporus cultivation. Microbiol. Ecol. 75:318-330.

Xia, F., Zhou, X., Liu, Y., Li, Y., Bai, X., and Zhou, X. 2019. Composition and predictive functional analysis of bacterial communities inhabiting Chinese cordyceps insight into conserved core microbiome. BMC Microbiol. 19:105.

Yang, W., Wang, L., Hu, Q., Pei, F., and Mugambi, M. A. 2019. Identification of bacterial composition in freeze-dried Agaricus bisporus during storage and the resultant odor deterioration. Front. Microbiol. 10:1-12.

Zarenejad, F., Yakhchali, B., and Rasooli, I. 2012. Evaluation of indigenous potent mushroom growth promoting bacteria (MGPB) on Agaricus bisporus production. World J. Microbiol. Biotechnol. 28:99-104. 